\title{
A Systematic Review of Gastrointestinal Manifestations in Diabetic Nephropathy
}

\author{
Shimin Zheng ${ }^{1,2}$ and Juan $\mathrm{Ma}^{1,2^{*}}$ \\ ${ }^{1}$ Department of Gastroenterology, Guangdong Provincial People’s Hospital/Guangdong Academy of Medical Sciences/Guangdong \\ Provincial Geriatrics Institute, Guangzhou, Guangdong, China; ${ }^{2}$ Medical College of Shantou University, Shantou,
}

Guangdong Province, China

\begin{abstract}
Diabetic nephropathy is the most common microvascular complication of diabetes mellitus. With the increasing prevalence of diabetes mellitus, diabetic neuropathy has become the primary cause of chronic nephropathy cases. Diabetes mellitus and chronic nephropathy exert certain effects on the digestive system. diabetic neuropathy patients also have digestive system symptoms and gastrointestinal diseases, with most presenting such problems as decreased gastrointestinal tract tension, insufficient gastric motility, decreased contractility, and delayed gastric emptying; these problems are often accompanied by symptoms of digestive system dysfunction, such as constipation, dry stool, tenesmus. This article will summarize and discuss the effects and mechanism of diabetic neuropathy involving the digestive system.
\end{abstract}

\section{Introduction}

Diabetes mellitus (DM) is a global epidemic, characterized by hyperglycemia. It is divided into type 1 diabetes mellitus (T1DM) and type 2 diabetes mellitus (T2DM), which account for $5-10 \%$ and $90-95 \%$ of DM cases resepectively. ${ }^{1}$ Diabetic nephropathy (DN) is one of the most common microvascular complications associated with DM, and $25-40 \%$ of DM patients may be at risk for $\mathrm{DN} .^{2}$ The disease is characterized by proteinuria and decreased glomerular filtration rate.

Most patients with diabetes and chronic kidney disease have symptoms involving the digestive system. Some studies have found that most patients with DN have similar digestive symptoms and gastrointestinal lesions. In DN, the degree of gastrointestinal lesions in advanced cases is higher than that in early cases. ${ }^{3} \mathrm{DN}$ patients often present with accompanying gastrointestinal tract tension and gastric motility deficiency, decreased contractility, delayed gastric emptying and other digestive system symptoms. ${ }^{4}$ Separately, patients with long-course T1DM under poor control can also present with gastrointestinal motility disorders, such as

Keywords: Diabetic nephropathy; Digestive system.

Abbreviations: CKD, chronic kidney disease; DD, diabetic diarrhea; DM, diabetes mellitus; DN, diabetic nephropathy; ESRD, end-stage renal disease; FC, functional constipation; FI, fecal incontinence; GER, gastroesophageal reflux; GLP1, glucagonlike peptide 1; GLUT2, glucose transporter type 2; GSRS, gastrointestinal Symptom Rating Scale; LPS, lipopolysaccharide; NAFLD, nonalcoholic fatty liver disease; $\mathrm{NASH}$, nonalcoholic steatohepatitis; PPI, proton pump inhibitors; SFL, simple fatty liver; T1DM, type 1 diabetes mellitus; T2DM, type 2 diabetes mellitus.

Received: May 29, 2019; Revised: July 29, 2019; Accepted: August 08, 2019

${ }^{*}$ Correspondence to: Juan Ma, 106 Zhongshan Second Road, Guangzhou, Guangdong Province, China, 510000. Tel: 18928857605; E-mail: mjlqh@163.com

How to cite this article: Zheng S, Ma J. A Systematic Review of Gastrointestinal Manifestations in Diabetic Nephropathy. Exploratory Research and Hypothesis in Medicine 2019;4(3):52-61. doi: 10.14218/ERHM.2019.00014 gastroparesis, constipation, diarrhea, and fecal incontinence. These symptoms are often associated with diabetic complications, such as autonomic neuropathy, peripheral neuropathy, and DN.

To gain a better understanding of the digestive symptoms caused by diabetic neuropathy in diabetes mellitus, we searched the PubMed database for publications related to "diabetic nephropathy" and "digestive system".

Pathogenesis of digestive system damage with diabetic nephropathy

\section{Intestinal barrier}

Destruction of the intestinal mucosal barrier often activates the adaptive immune response and leads to a local or systemic inflammatory response. Endotoxemia and systemic inflammation caused by intestinal mucosal barrier defects are risk factors for cardiovascular diseases. Increased creatinine production and reduced renal clearance in patients with chronic kidney disease (CKD) are likely to lead to accumulation of inflammatory factors in the body. Animal experiments have shown that CKD features intestinal mucosal barrier dysfunction. ${ }^{5}$ The inflammatory state in the development of DM and CKD may be closely related to intestinal mucosal barrier. Firstly, the transport of bacterial and bacterial products, such as lipopolysaccharide (LPS), from the intestinal cavity to the blood that is caused by increased intestinal permeability can aggravate the low inflammation state of T2DM, CKD, and end-stage renal disease (ESRD), i.e. "gut leakage syndrome". ${ }^{6}$ Secondly, changes in species richness, diversity, composition and function of intestinal microflora may have a profound impact on intestinal physiology by altering nutritional utilization and biosynthesis of bioactive metabolites. $^{7}$ 
Intestinal epithelial cells participate in mechanical protection of the mucosal barrier via their tight junctions, preventing the transfer of intestinal chemicals and microorganisms from the intestine to the blood along the paracellular pathway. The tight junction is the main determinant of intestinal barrier permeability. The impairment of intestinal barrier permeability leads to the transport of intestinal contents to deep tissues, activating adaptive immune response and local or systemic inflammation.

The destruction of normal intestinal mucosal barrier may promote bacterial endotoxins from entering the circulation. Studies have shown that tight junction proteins in intestinal epithelial cells of CKD rat models are significantly reduced. Furthermore, incubation of human intestinal epithelial cells with uremic plasma can increase their permeability and decrease the expression of tight junction protein. ${ }^{5}$ Urea is one of the factors known to lead to the destruction of epithelial tight junctions in CKD. Urea diffuses from the blood to the intestinal cavity and is metabolized to ammonia by intestinal bacterial urease, after which it is converted to ammonium hydroxide, which can destroy tight junction proteins in epithelial cell spaces. ${ }^{8}$

Glucose is considered to be the coordinator of the intestinal mucosal barrier. Glucose transport between intestinal epithelium and systemic circulation is mediated by glucose transporter type 2 (GLUT2). ${ }^{9}$ Christoph et al. ${ }^{10}$ suggested that hyperglycemia significantly interferes with the integrity of homeostasis epithelium, resulting in abnormal influx of immunostimulatory microbial products and systemic transmission of intestinal pathogens. Hyperglycemia leads to retrograde transport of glucose to intestinal epithelial cells via GLUT2, resulting in changes in intracellular glucose metabolism, eventually leading to mucosal barrier dysfunction and microbial translocation to the systemic circulation. One of the most important pathways affected by hyperglycemia is glycosylation of protein $\mathrm{N}$ in endoplasmic reticulum and the Golgi apparatus, which may be key to the regulation of various epithelial functions. ${ }^{11}$ After insulin treatment, insulin can prevent the destruction of tight junction integrity and the transfer of microbial products. Glucose-mediated metabolic changes in epithelial cells lead to impaired intestinal mucosal barrier function, which reduces the host's defense against intestinal infection. In conclusion, a series of experiments have confirmed that hyperglycemia directly affects intestinal epithelial cells, which can be an important cause of intestinal mucosal barrier dysfunction. ${ }^{10}$

\section{Intestinal microorganisms}

Human intestinal microflora is a complex ecosystem, being interdependent with the health of a host, protecting organisms from pathogens, regulating the internal metabolism of the immune system and carbohydrates and lipids, and contributing to nutritional balance. The number of microorganisms varies along the intestinal tract, with the highest content being in the distal intestine. ${ }^{12}$ Intestinal microflora plays an important role in nutrition and protection in vivo. It plays a certain role in food digestion, vitamin biosynthesis, bile acid biotransformation, innate immunity and gut barrier maintenance. Intestinal microflora produce short-chain fatty acids, such as acetic acid, propionic acid and butyric acid, by fermenting undigestible carbohydrates. This fermentation product is an important energy source for colon cells, which can reduce the intestinal $\mathrm{pH}$ value and help maintain the ideal environment of homeostasis in the colon. ${ }^{13}$

Intestinal microorganisms maintain intestinal functional integrity by repairing tight junction protein structure, inducing epithelial heat shock protein, up-regulating the mucin gene, and com- peting with pathogenic bacteria for intestinal epithelial binding sites. ${ }^{14}$ Many studies have found that there are significant changes in the composition of intestinal microflora in T1DM patients. ${ }^{15}$ The changes of intestinal microflora are closely related to the decrease in glucose tolerance. The number of butyric acid-producing bacteria in intestinal microflora of DM patients decreases, while the number of pathogenic bacteria increases. Butyrate is the main energy source of intestinal epithelium. It can increase the secretion of glucagon-like peptide 1 (GLP1) and casein (PYY) by colon L cells, ${ }^{16}$ and increase the intestinal transit time. ${ }^{17}$

Intestinal microflora can change with the biochemical environment. Urea is a key factor in increasing intestinal barrier permeability in CKD. Microorganisms play an important role in ammonia and urea metabolism. Taking the urea nitrogen recovery pathway as an example, the final product of protein catabolism in mammals is ammonia, which becomes toxic to cells at high concentration. Under normal circumstances, ammonia is converted into urea through the ornithine-urea cycle in the body, and intestinal microorganisms further decompose urea into ammonia and carbon dioxide. In uremic patients, the increase of urea inflow into the intestinal cavity leads to the expansion of urea-decomposing bacteria and the increase of ammonia production, which results in increased intestinal $\mathrm{pH}$ and further evolves into mucosal irritation and structural damage. Similarly, increased intestinal nitrogen excretion in CKD patients will also lead to overgrowth of microorganisms using these substrates. ${ }^{18}$

\section{Helicobacter pylori}

H. pylori infection is directly or indirectly related to many digestive diseases. About $50 \%$ of the world's population is infected with H. pylori, and the infection rate in some developing regions is even more than $70 \% .{ }^{19}$ Diabetic gastroparesis is due to decreased gastric motility caused by autonomic neuropathy, which leads to prolonged food retention time and continuous stimulation of $\mathrm{G}$ cells to secrete gastrin, thereby increasing gastric acid secretion and causing gastric mucosal injury. This mechanism makes advanced DN patients more susceptible to $H$. pylori infection. This may also partly explain the occurrence of digestive tract symptoms in patients with DN. Duodenal fluid reflux after pylorus relaxation also results in gastric mucosal barrier damaged by reflux bile, making gastric mucosa more sensitive to $H$. pylori growth.

In 1998, Simon et al. ${ }^{20}$ were the first the report on the relationship between H. pylori and DM. They had found that the prevalence of H. pylori in DM patients was higher than that in the control group in their study. However, some scholars have since obtained different results. Compared with nondiabetic control groups, the prevalence of $H$. pylori in DM patients has been similar or even lower. ${ }^{21,22}$ Zhou et al. ${ }^{23}$ showed that the prevalence of $H$. pylori infection in T2DM patients with microalbuminuria and massive albuminuria was significantly higher than that in normal albuminuria and nondiabetic control groups, suggesting that $H$. pylori infection is associated with T2DM. In addition, that study showed that the prevalence of $H$. pylori infection was higher in T2DM patients with DN than in T2DM patients without renal complications. DM patients are prone to secondary chronic infection, and delayed gastric emptying caused by diabetic gastroparesis in $\mathrm{DN}$ patients may also lead to overgrowth of bacteria in the upper gastrointestinal tract, which is considered to be the root cause of H. pylori infection.

Although the experiments by Zhou et al. ${ }^{23}$ showed that the prevalence of $H$. pylori infection in patients with DN was increased, endoscopy results showed that there was no significant difference 
in the detection rate of gastroduodenal mucosal lesions among the three groups of DM patients, DN patients, and nondiabetics. ${ }^{23}$ The etiology of digestive system diseases is complex and diverse. The relationship between DN and gastroduodenal morbidity still needs to be further explored.

H. pylori may play a role in promoting the development of DN. Studies have shown that microalbuminuria in $H$. pylori-positive T2DM patients is significantly higher than that in H. pylori-negative T2DM patients and urinary protein levels in $\mathrm{H}$. pylori-positive DN patients are significantly higher than those in H. pylori-negative DN patients. ${ }^{24,25}$ Proteinuria is known to be a risk factor for DN progression, but there are few studies on it and H. pylori infection. ${ }^{26}$ The exact mechanism remains to be further explored.

\section{Gastrointestinal hormones}

Gastrointestinal hormones are mainly produced by endocrine cells of the gastrointestinal mucosal epithelium. Gastrointestinal motility is regulated by nerves and hormones. Gastrointestinal hormones play an important role in regulating feeding behavior and gastrointestinal motility. Dyspepsia often exists in patients with DN, which may be associated with gastrointestinal dysfunction, decreased motilin activity, and weakened gastrointestinal motility, which cause the feedback that the motilin secretion increases to regulate gastrointestinal motility in patients with diabetic gastroparesis.

The causes of digestive system symptoms in DN are various. Gilbey et al. ${ }^{27}$ believes that the pathogenesis of gastrointestinal lesions in $\mathrm{DN}$ is related to peripheral autonomic neuropathy, gastrointestinal smooth muscle degeneration, hyperglycemia, and degradation of gastrointestinal peptide hormones. Long-term history of DM and long-term hyperglycemia can lead to elevated intracellular sorbitol levels and decreased inositol levels, resulting in neuronal degeneration and segmental demyelination of axons. At the same time, long-term hyperglycemia can inhibit gastrointestinal motility and increase gastrin, resulting in secondary abnormal motilin secretion and gastric motility, and eventually lead to gastric rhythm disorder and prolonged gastric emptying time. ${ }^{28}$ Diabetic microangiopathy can cause ischemia, neurotrophic impairment and loss, aggravate smooth muscle lesions, and cause dysfunction.

The kidney is the key site for degradation and removal of gastrointestinal peptide hormones. DN is often accompanied by kidney disease. When renal insufficiency occurs, the impaired degradation process can lead to internal environment disorders and abnormal gastrointestinal hormones. Studies have shown that serum gastrin, motilin and glucagon levels in patients with stage III and IV DN are higher than those in patients with stage I and II, and gastric emptying time is prolonged. This suggests that the degree of renal dysfunction is closely related to the decline of gastrointestinal function. It is speculated that the degree of renal dysfunction can be predicted by detecting the changes of gastrointestinal hormones in patients with DN. ${ }^{29}$

\section{Uremic toxins}

DN is often accompanied by renal insufficiency and elevated urea levels, and can ultimately develop into uremia. The plasma of uremic patients contains more than 200 metabolites or toxic substances, collectively known as uremic toxins. Uremic toxins can be derived from the accumulation of metabolites in vivo, or from the excretion of exotic toxicants without detoxification, the production of toxic metabolites or the continuous increase of normal physiological active substances. Common uremic toxins, such as urea and guanidine compounds, can cause nausea, vomiting, and other gastrointestinal symptoms. Ammonia produced by the degradation of urea in vivo by intestinal microorganisms can change the intestinal $\mathrm{pH}$ value.

Recent studies have shown that azotemia has certain effects on intestinal microorganisms and the intestinal mucosal barrier, and can cause gastrointestinal dysfunction and even extensive mucosal damage. ${ }^{20}$ Studies have also shown that CKD patients with high urea level are prone to upper gastrointestinal symptoms. Most of the biopsies show mucosal lesions, such as erosive gastritis, esophageal ulcer, and duodenitis. ${ }^{30}$ The possible mechanism is that the increase of urea causes abnormal intestinal barrier function, including the destruction of tight junction proteins and intestinal flora imbalance, which may cause damage to the gastrointestinal mucosa.

In $\mathrm{CKD}$, the increase in urea leads to the reverse diffusion of hydrogen ions across the mucosal barrier, whereby the clearance rate of gastrin in the kidney decreases and the synthesis increases, leading to the increase of gastrin level. High gastrin levels in patients with DN can reduce pyloric sphincter tension, which may lead to bile reflux worsening mucosal damage. The retention of guanidine compounds can interfere with nerve activity, excite the parasympathetic nerve, and destroy the stability of the internal environment In addition, uremic toxins also impair pancreatic exocrine function (Fig. 1). ${ }^{31}$

Diabetic nephropathy-related pharyngeal and esophageal diseases

Abnormal pharyngeal and esophageal motility is often found in DM patients, and is more common in patients with peripheral or autonomic neuropathy. DM patients have abnormal esophageal motility, such as delayed esophageal emptying, which is more common in patients with DN. Delayed esophageal transport usually reflects decreased peristalsis or foci of low esophageal pressure, but patients may not have esophageal symptoms.

Gastroesophageal reflux (GER) may also exist in some patients with T1DM. In patients with symptomatic diabetic gastroparesis, a combination of gastric dilatation and increased residual gastric volume may increase the risk of GER. In DM patients, the lower esophageal sphincter is usually functioning normally. In DN, dyskinesia of the upper gastrointestinal tract and dystonia of the pyloric sphincter, delayed gastric emptying and rigidity of upper gastrointestinal tract caused by diabetic gastroparesis may represent the mechanisms leading to pathological GER in patients with DN. ${ }^{32}$

\section{Diabetic nephropathy-related gastropathy}

Diabetic stomach disease is considered to be a syndrome of gastric dysfunction associated with DM.

\section{Gastroparesis}

Gastroparesis is one of the common chronic complications of both T1DM and T2DM. It is characterized by insufficient gastric motility and gastric emptying disorder. Without gastric mechanical obstruction, delayed emptying of solid food in the gastric cavity in patients with gastroparesis results in early satiety, postprandial fullness, nausea, vomiting, belching, and abdominal distention. ${ }^{33}$ Some DM patients have pyloric spasm, which leads to obstructive 


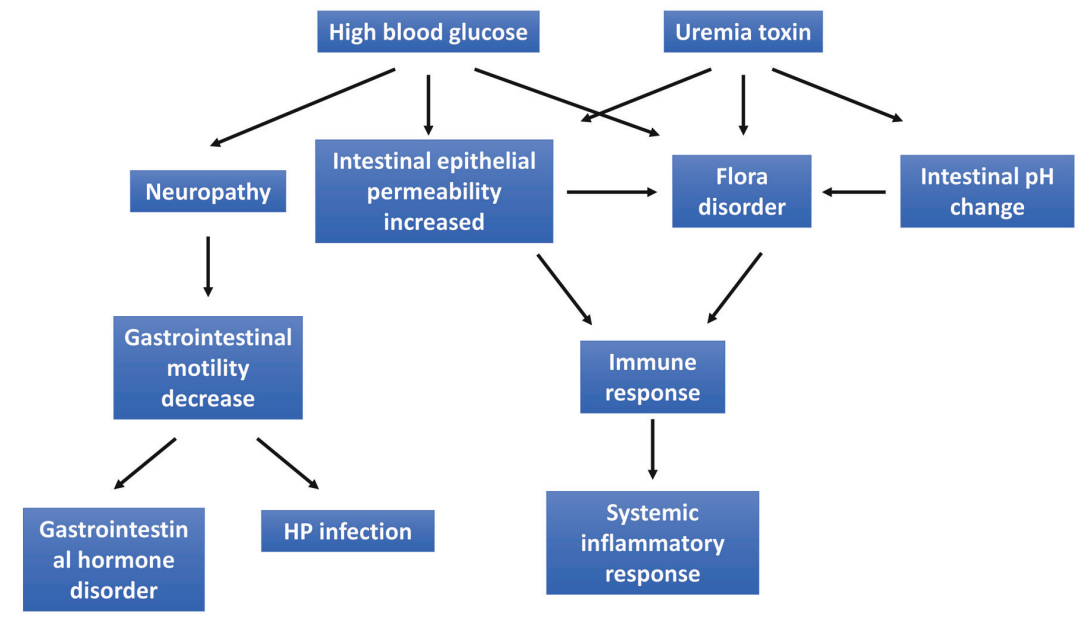

Fig. 1. The main mechanism of diabetic nephropathy effect on the digestive system.

gastroparesis. Some scholars have proposed that gastroparesis is more common in patients with DN and that there is a significant correlation between them. ${ }^{34}$

$\mathrm{DN}$ is a risk factor for delayed gastric emptying. ${ }^{35}$ Moreover, patients often have delayed gastric emptying due to retention of gastric contents. Similar phenomena often occur in patients with end-stage renal failure. Pyloric function is also one of the determinants of gastric emptying. Nausea and vomiting in some DM patients are associated with pyloric spasm. Whether activated by central or peripheral mechanisms, vomiting can cause gastric slow wave arrhythmia, inhibit antral contraction and small intestinal motility, and eventually cause gastric emptying disorders.

\section{Gastric diseases without delayed emptying}

DM patients without delayed gastric emptying can also have symptoms of stomach diseases, such as nausea and vomiting. Some patients even have accelerated gastric emptying. The condition may be the result of impaired gastric receptive relaxation and regulatory response due to abnormal autonomic innervation in the fundus of the stomach. Physiological factors, such as decreased proximal gastric compliance, may be responsible for increased gastric distension in patients with T1DM. In addition, the concentration of blood glucose can also affect gastrointestinal sensation. Gastric distension in hyperglycemia can produce stronger nausea and fullness. In addition, nausea and vomiting often occur in acute ketoacidosis of DM, which can be alleviated by treatment.

\section{Upper gastrointestinal hemorrhage}

Upper gastrointestinal hemorrhage refers to bleeding of the digestive system above the flexor ligament, and includes lesions of the esophagus, stomach, duodenum, pancreas, and gallbladder. It appears as melena when the amount of bleeding is small, and may be accompanied by hematemesis or even peripheral ischemia when the amount of bleeding is large. Studies have shown that DM is a risk factor for upper gastrointestinal hemorrhage. ${ }^{36} \mathrm{~A}$ study collected 50 cases of uremic deaths and analyzed the causes. The results showed that nearly half of the cases had DN, among which $22 \%$ were caused directly by gastrointestinal hemorrhage. ${ }^{37}$

The causes of upper gastrointestinal hemorrhage in patients with DN are various. In diabetic hyperglycemia, the increase of glycosylated hemoglobin leads to the relative decrease of oxidized hemoglobin and the obvious decrease of tissue oxygen uptake, resulting in chronic hypoxia of gastric mucosa. When gastric mucosa ischemia and hypoxia occur, energy metabolism will be impaired and gastric mucosal barrier function will be destroyed, resulting in extensive congestion, edema, erosion and hemorrhage under the action of pepsin and hydrogen ions. In addition, when the secretion of insulin-antagonistic hormones, such as adrenaline, increases in DM patients, blood glucose increases further. Under hypoxic stress, the secretion of stress hormones like catecholamine can also lead to damage of the gastric mucosal barrier and gastric mucosal stress ulcers. $^{38}$

Due to renal dysfunction, metabolic waste of DN remains in the digestive system. These metabolic waste materials, such as urea, amines and guanidine, can induce or aggravate the symptoms of upper gastrointestinal hemorrhage through various biochemical mechanisms. DN or DM with hepatic insufficiency can lead to coagulation dysfunction and aggravate gastric mucosal bleeding. Other factors include gastroparesis, distal esophageal tear because of pyloric spasm and severe vomiting, high susceptibility to $H$. pylori in patients with $\mathrm{DN}$, or hypersplenism in patients with $\mathrm{DM}$, and impaired liver function, which can lead to gastroduodenal ulcer and upper gastrointestinal hemorrhage. Upper gastrointestinal hemorrhage caused by DN is rare and acute. It is an important measure to reduce the risk of upper gastrointestinal hemorrhage by strengthening the study of its pathogenesis, early diagnosis and early use of gastric mucosal protective agents or proton pump inhibitors.

\section{Diabetic nephropathy-elated intestinal diseases}

\section{Small intestinal diseases}

DM may promote the occurrence of intestinal mucosal injury, especially T2DM, which is associated with the severe injury. DM can cause intestinal mucosal inflammation, motility disorder, flora imbalance, permeability increase, microcirculation disturbance, circulation change, and intestinal stem cell dysfunction. Small intestinal injury can lead to intestinal erosion, ulcer, bleeding, and even perforation with serious consequences. Some scholars have used capsule 
endoscopy to collect data on 38 cases of T2DM and 152 cases of non-DM. They recorded and analyzed the situation of small intestinal mucosal injury. The results showed that T2DM cases had more small intestinal villus edema and higher Lewis score of small intestinal mucosal injury, especially for those with DN, suggesting that DN supported the development of intestinal mucosal injury. ${ }^{39}$

\section{Colon diseases}

There is also a correlation between DN and colon disease. Studies have shown that patients with T1DM and ESRD have abnormal intestinal mucosal morphology, such as reduced numbers of intestinal epithelial cells and crypts, which may be related to the destruction of colonic stem cells. ${ }^{36}$ The relative risk of colorectal cancer in patients with DM is increased, which may be related to insulin resistance, diet and poor lifestyle in patients with T2DM. DN is also associated with an increased incidence of colorectal cancer, and the mortality rate of it also increases. ${ }^{40}$ Increased mortality of colon cancer has also shown a consistent trend with increased proteinuria. $^{41}$

\section{Diabetic diarrhea}

Diabetic diarrhea (DD) is a manifestation in the digestive system during the middle and late stages of DM development. It often manifests as refractory intermittent diarrhea. The stools are brown-yellow and water-like, with a large amount and often accompanied by fat diarrhea and severe sensation of tenseness. The diarrhea often occurs at night and in the early morning. Laboratory examination is often normal in these cases upon clinic presentation. Barium meal test of the digestive system shows a normal shape of the small intestine, but the barium passes faster or longer. Colonoscopy shows normal intestinal mucosa or congestion and edema. The diagnostic criteria of DD are as follows: 1 . obvious diarrhea symptoms with incomplete stool, no abdominal pain, no abnormal stool routine, and poor drug treatment effect; 2. long history of DM; 3. hypoglycemic drugs effectively alleviating abdominal pain symptoms; and 4. exclusion of other diseases that may cause diarrhea.

Diabetic autonomic neuropathy can lead to a decrease in intestinal motility, slow intestinal peristalsis, and prolonged transmission time. Reduction of intestinal Cajal cells can also lead to diabetic intestinal dyskinesia. The secretion of gastrointestinal hormones in DM patients is often disturbed. Diarrhea is prone to occur when the hormone effect of promoting gastrointestinal motility is stronger than that of inhibiting gastrointestinal motility. The pancreatic exocrine function of DM patients is often disturbed to varying degrees, which can lead to poor fat absorption and pancreatic diarrhea. ${ }^{42}$ Diabetic autonomic neuropathy can lead to gallbladder nerve involvement and biliary diarrhea. Diabetic autonomic neuropathy can also involve the patient's rectoanal sphincter, which leads to fecal incontinence and increased frequency, and can also make the rectal sensory function decline, leading to diarrhea. Bacterial imbalance and intestinal malabsorption may also be the reason of diarrhea caused by DN. ${ }^{43}$

\section{Anorectal dysfunction}

Diabetic autonomic neuropathy can lead to sympathetic and vagal nerve tension imbalance that innervates the rectal and anal sphincter, weaken the tension of the anal sphincter, impair the function of the rectal and anal sphincter, and decrease the rectal sensory function of patients, resulting in fecal incontinence, fecal frequency increase, defecation effort and other manifestations of anorectal dysfunction. Some scholars used the Gastrointestinal Symptom Rating Scale (GSRS) to evaluate patients with long-term T1DM and ESRD. The intestinal symptoms including diarrhea, abdominal pain and constipation have been found to be related to anorectal sphincter dysfunction. ${ }^{44}$

Fecal incontinence (FI)

FI is defined as unconscious loss of solid or liquid feces. Gastric paralysis, constipation, diarrhea, and fecal incontinence often occur in patients with long-term and poorly controlled T1DM. Abnormal gastrointestinal motility in these patients is often associated with autonomic neuropathy and other diabetic complications, such as peripheral neuropathy, nephropathy, and retinopathy. In community surveys, intestinal disorders, especially diarrhea and rectal emergencies, and chronic diseases, such as DM, are the important independent risk factors for FI identified so far. ${ }^{45}$ Hyperglycemia can inhibit the movement of the external anal sphincter, reduce rectal compliance, and increase the risk of FI. Long-term hyperglycemia can lead to decreased tension of the anal sphincter, which can lead to FI and other complications.

\section{Constipation}

Constipation is characterized by fecal frequency reduction (fewer than 3 times per week), dry stool, and/or difficulty in defecation. Difficulties in defecation include laborious defecation, incomplete defecation, time-consuming defecation, and the need for manualassistance. Referring to the Roman IV standard, functional constipation (FC) is a kind of functional bowel disease. The diagnostic criteria are as follows (i.e. must include two or more of the items): 1. more than $25 \%$ of the excreta feels laborious; 2 . more than $25 \%$ of the excreta is dried ball or hard dung; 3 . more than $25 \%$ of the excreta is accompanied by a sense of incompleteness; 4 . more than $25 \%$ of the excreta is accompanied by a sense of anorectal obstruction or obstruction; 5 . more than $25 \%$ of the excreta feels laborious; 6 . more than $25 \%$ of defecation needs manual assistance (such as finger-assisted defecation, pelvic floor support); 7. spontaneous defecation occurs less than three times a week; 8. when laxatives are not used, feces rarely appear; 9 . the condition does not meet the diagnostic criteria of IBS. Symptoms must appear at least 6 months before diagnosis, and symptoms in the recent 3 months must meet the above diagnostic criteria. ${ }^{46}$

While most studies have shown that there is a positive correlation between DM and constipation symptoms, the most recent studies have shown that there is no significant difference between DM and intestinal symptoms (including constipation and diarrhea) ${ }^{47} \mathrm{DM}$ patients show more chronic gastrointestinal symptoms, and the risk of constipation, dry stool, incomplete bowel movement and rectal pressing symptoms increases, even after excluding gastrointestinal organic lesions. DN may increase the risk of these symptoms. ${ }^{4}$

Diabetic nephropathy-related liver diseases

\section{Fatty liver}

Nonalcoholic fatty liver disease (NAFLD) is defined as acquired 
metabolic stress liver injury, including simple fatty liver (SFL), nonalcoholic steatohepatitis (NASH) and related cirrhosis. The disease condition is closely related to IR and genetic susceptibility, due to increased fat storage in the liver in the form of triglycerides (more than $5 \%$ of the weight of the liver), excluding excessive drinking or other specific liver-damaging factors. A large number of studies have confirmed that DM and NAFLD have a significant correlation, and that they affect each other.

Diabetic liver disease is usually the result of steatosis of the liver. The prevalence and severity of NAFLD in DM patients are increasing. DM is an independent risk factor for the development and progression of NAFLD to advanced liver diseases, including liver fibrosis, cirrhosis, and hepatocellular carcinoma. Compared with non-DM patients, patients with T2DM have an increased risk of NAFLD and a higher risk of liver fibrosis and cirrhosis.

Increasing fasting insulin and hepatic IR may lead to the decrease of hepatic lipoprotein metabolism and the increase of very low density lipoprotein synthesis, resulting in the increase of intrahepatic fat production and the accumulation of fatty acids from other organs in the liver. IR is a major risk factor for NASH. ${ }^{48}$ IR increased significantly in NASH patients. A cross-sectional study of 188 subjects examined the effect of steatohepatitis severity on atherogenic dyslipidemia in patients with NAFLD. The results showed that the IR of NAFLD patients was significantly higher than that of non- NAFLD patients, regardless of obesity or nonobesity condition. ${ }^{49}$ Therefore, IR may be one of the pathogenic mechanisms of NAFLD, and there is a positive correlation between IR and renal insufficiency. ${ }^{50}$ Data show that the incidence of renal insufficiency in NAFLD patients is significantly higher, and there is a correlation between liver fat accumulation and renal insufficiency. ${ }^{51}$

The relationship between DN and NAFLD is also very close, and there may be a mutually reinforcing relationship between them. A retrospective study has confirmed that NAFLD is a risk factor for DN, and increased liver fat content may be associated with increased damage to DN. ${ }^{52}$ The glomerular filtration rate of NASH patients is decreased,$^{53}$ and the risk of proteinuria in them is increased significantly. ${ }^{54}$ With the increase of liver fat content, the cumulative rates of proteinuria and microalbuminuria increases, and the glomerular filtration rate further decreases. ${ }^{53}$ In Chinese patients with T2DM, advanced hepatic fibrosis is a serious form of NAFLD, which is independently related to the high risk of proteinuria, that is, the risk of proteinuria increases with the severity of hepatic fibrosis. ${ }^{55} \mathrm{DN}$ also affects NAFLD.

Patients with T2DM have significantly increased prevalence of NAFLD. ${ }^{56}$ Sixteen patients with end-stage DN were treated with intraperitoneal or subcutaneous insulin during continuous ambulatory peritoneal dialysis, and their liver fat accumulation was assessed. The results showed that intraperitoneal insulin combined with glucose-based peritoneal dialysate induced subcapsular fatty degeneration of liver. When the peritoneal metastasis rate and body weight increased, the content of subcapsular fatty degeneration increased. ${ }^{57} \mathrm{~A}$ total of 933 subjects, including 530 patients with hepatic steatosis, were enrolled in another study to investigate whether the presence of hepatic steatosis and NAFLD is associated with decreased renal function or microalbuminuria in patients with T2DM. The results showed that 388 patients with hepatic steatosis had NAFLD and both hepatic steatosis and NAFLD were significantly correlated with the rate of renal function decline. ${ }^{55}$ This suggests that $\mathrm{DN}$ has certain effects on NAFLD. However, some scholars have put forward different views ${ }^{58}$ Nevalainen et al. ${ }^{57}$ carried out an experiment that included 413 patients with T2DM and they found that the presence of NAFLD was not related to DN. The relationship between DN and
NAFLD needs further study.

\section{Hepatocellular carcinoma}

There is a significant correlation between DM and hepatocellular carcinoma, and individuals with DM have a higher risk of developing hepatocellular carcinoma. The 2012 meta-analysis of 18 cohort studies showed that people with DM had a 2-fold increased risk of developing hepatocellular carcinoma compared with non-DM patients, and this significant association was not associated with alcohol or viral hepatitis status. ${ }^{59}$ Mild steatosis and liver inflammation may progress to fibrosis and hepatocellular carcinoma. T2DM can increase liver fibrosis by a factor of three and hepatocellular carcinoma by a factor of two. ${ }^{60} \mathrm{IR}$ and subsequent inflammatory cascades may trigger the oncogenic potential of NASH. IR also leads to increased release of various proinflammatory cytokines, including tumor necrosis factor- $\alpha$, interleukin- 6 and leptin, which contribute to the development of hepatic steatosis, inflammation, and the subsequent cancer.

The related mechanisms of the impact of DM on the risk of hepatocellular carcinoma have not yet been fully elucidated. There are several hypotheses, though. First, DM is one of the risk factors for the development of NAFLD. In early T2DM, hyperinsulinemia may up-regulate the insulin-like growth factor-1 (IGF-1). IGF-1 stimulates cell proliferation and inhibits apoptosis in the liver. In vitro studies, such as those with animal models, and epidemiology also support the involvement of insulin and IGF-1 in the development of hepatocellular carcinoma. ${ }^{61}$ The risk of hepatocellular carcinoma may also be affected during DN. Targher et al. ${ }^{62}$ suggested that possible molecular mediators linking NAFLD to CKD may include increased release of certain pathogenic mediators in the liver, such as advanced glycation end-products, reactive oxygen species, C-reactive protein, tumor necrosis factor- $\alpha$ and interleukin-6, which may be one of the causes of hepatocellular carcinoma in DN.

Diabetic nephropathy-related biliary diseases

\section{Gallbladder emptying}

Diabetic autonomic neuropathy can cause gallbladder nerve involvement, affect gallbladder movement, cause regular bile excretion disorder, and lead to biliary diarrhea. Compared with normal people, DM patients have larger fasting gallbladder volume and lower biliary excretion fraction, leading to biliary dysfunction, ${ }^{43}$ which can be the reason for DM patients experience biliary dysfunction.

The causes of gallbladder emptying disorder in DM patients are various. First of all, diabetic autonomic neuropathy causes gallbladder nerve involvement, affects the regular excretion of bile, and leads to delayed gallbladder emptying. Second, gastroduodenal emptying during digestive and interdigestive periods can increase gallbladder emptying, and gallbladder emptying can promote duodenal emptying, thereby promoting gastric emptying, while gastrointestinal motility disorders can cause gallbladder emptying disorders. DM is often accompanied by low gastrointestinal motility, such as gastroparesis and gastric retention, which can lead to gallbladder emptying disorders. In addition, DM patients often have Oddi's sphincter dysfunction due to autonomic neuropathy. The main manifestation is increased tension of the Oddi's sphincter in the fasting state, resulting in increased pressure in 
the common bile duct, bile reflux into the gallbladder, and resulting in gallbladder filling and cholestasis. ${ }^{63}$ Gastrointestinal hormone secretion disorders often occur in DM patients. When the hormone effect of inhibiting gallbladder movement is stronger than that of promoting gallbladder movement, gallbladder emptying will be affected, resulting in cholestasis. ${ }^{64}$

\section{Gallstones}

Gallstone is often clinically characterized by pain in the right upper abdomen and sometimes radiated to the right shoulder and back, accompanied by nausea, vomiting and other gastrointestinal discomfort symptoms. The incidence of gallstones in patients with T2DM is increased significantly. Agunloye et al. ${ }^{65}$ found that the incidence of gallstones in patients with T2DM was closely related to the course of DM, through abdominal ultrasonography in 400 patients with T2DM. Toosi et al. ${ }^{66}$ also proposed that the prevalence of gallstones was positively correlated with the course of DM. Most patients with DM complicated with gallstones have no obvious discomfort symptoms. Some patients have mild right upper abdominal distension, discomfort, indigestion, and other phenomena. When the cholecystic duct or common bile duct is blocked by stones, gallstones can easily cause gallbladder colic or acute cholecystitis due to stimulation of the gallbladder mucosa or blockade in the gallbladder duct. Severe pain in the right upper abdomen, fever, nausea, vomiting and even gallbladder perforation and necrosis can occur. ${ }^{63}$

\section{Diabetes-related pancreatic diseases}

\section{Pancreatic exocrine function}

The pancreas of DM patients often suffers from exocrine dysfunction to varying degrees. The related factors of pancreatic exocrine dysfunction associated with T1DM and T2DM are different. Pancreatic exocrine dysfunction in patients with T2DM may be related to body mass index and pancreatic artery lesions, while T1DM may be related to the course of DM and glutamate decarboxylase antibody. ${ }^{67} \mathrm{DM}$ patients with pancreatic exocrine dysfunction may have diarrhea, fat diarrhea, weight loss, and other clinical manifestations. Trypsin supplementation therapy can effectively improve their symptoms. ${ }^{68}$

There are several possible mechanisms of diabetes-related pancreatic exocrine dysfunction. These include diabetic autonomic neuropathy leading to impaired intestinal nerve reflex, diabetic microangiopathy leading to insufficient perfusion of pancreatic exocrine tissue and local ischemia, leading to pancreatic fibrosis, insulin impairing the regulatory function of pancreatic exocrine tissue, and lack of local high-concentration insulin nutrition and increase of insulin's antagonistic hormones, such as glucagon, resulting in partial atrophy of pancreatic exocrine tissue. ${ }^{69}$

\section{Pancreatitis}

Pancreatitis is a disease of the pancreas, caused by the self-digestion of trypsin. The pancreas can be edema, congestion, or hemorrhage and necrosis. Clinical symptoms include abdominal pain, abdominal distension, nausea, vomiting, and fever. Recent studies have shown that DM itself is one of the risk factors of acute pancreatitis. Pancreatitis can aggravate DM, while DM can aggravate the degree of pancreatic infection. Infection and elevated blood glucose effect each other. Increased blood glucose is closely related to the onset of severe acute pancreatitis, and is one of the risk factors for poor prognosis. Compared with non-DM patients, the incidence of acute pancreatitis in patients with T2DM was significantly higher. ${ }^{70}$

\section{Pancreatic cancer}

Pancreatic cancer has high invasiveness, rapid development, poor prognosis and high fatality. The 5 -year survival rate of pancreatic cancer is less than $1 \%$. Studies have shown that DM patients are more susceptible to pancreatic cancer. ${ }^{71}$ A study of 111 cases of pancreatic cancer found that nearly one third of pancreatic cancer patients met the diagnostic criteria for DM. ${ }^{72}$ It has also been reported that more than half of the patients with pancreatic ductal cancer have DM or hyperglycemia, and $20-25 \%$ of the patients develop DM 6-36 months before the diagnosis of pancreatic cancer. DM with poorly controlled blood glucose gives an increased risk of pancreatic cancer. ${ }^{73} \mathrm{DM}$ with an increase in glycosylated hemoglobin of more than $4 \mathrm{mmol} / \mathrm{L}$ is an independent risk factor for pancreatic cancer. ${ }^{74}$ Finally, DM with pancreatic cancer is more common in elderly patients, and in males versus females. Many patients have symptoms of upper abdominal pain, abdominal distention, and poor appetite. A few patients may have jaundice and other symptoms. ${ }^{75}$

The effect of medications for treating digestive system diseases on blood glucose level and renal function

Digestive system medication also has a certain effect on blood glucose level and renal function.

\section{Gastric stimulants}

Mosapride, a gastric motility drug, is a selective agonist of the 5-HT4 receptor. It may improve insulin sensitivity in T2DM by increasing the secretion of GLP-1. The mechanism of its improvement on hyperglycemia is unclear. ${ }^{76}$ Liraglutide, as a long-acting analogue of GLP-1, can not only increase satiety and improve gastrointestinal emptying disorder but also regulate insulin levels. It has a positive intervention effect on DN in different stages. ${ }^{77}$

\section{Proton pump inhibitors (PPI)}

Pantoprazole, a PPI, can significantly reduce the level of glycosylated hemoglobin in T2DM. ${ }^{78}$ PPI can decrease gastric acid and indirectly increase serum gastrin level through a negative feedback effect. This may stimulate the proliferation and function of pancreatic $\beta$ cells and improve blood glucose control. Studies of a small number of T2DM patients have shown that glycosylated hemoglobin in patients taking PPI is slightly lower than that in non-PPI users. In patients with T2DM, the glycosylated hemoglobin levels in both groups were improved, but the addition of a PPI did not affect blood glucose control. ${ }^{76}$

Gastrin may increase the quality of pancreatic $\beta$ cells and improve glucose tolerance. Some studies have shown that PPI have a positive effect on blood glucose, especially in patients with T2DM. The mechanism may be related to the elevation of serum gastrin level. Some experiments have further suggested that the combina- 
tion of gastrin or PPI and a GLP-1 receptor agonist has a beneficial effect on blood glucose control in animal models. ${ }^{79}$ It was pointed out that in patients with T2DM, the glycosylated hemoglobin level of patients taking a PPI was slightly lower than that of the control group. In one study, the glycosylated hemoglobin levels of both groups were improved, but the addition of a PPI did not affect blood glucose control. ${ }^{76}$

In addition to the effect on blood glucose, PPI also affects renal function, which may be related to renal dysfunction. A large population-based study found a relationship between PPI use and the risk of acute interstitial nephritis and acute kidney injury in children. $^{80}$

\section{Gastric mucosa protectant}

Bismuth is a commonly used gastric mucosal protective agent. Taking toxic doses of bismuth will lead to acute oliguric renal failure. The mechanism may be that high doses of bismuth make cell membranes unstable and induce cell death through necrosis in vivo and in vitro. ${ }^{81}$

\section{Treatment of digestive system diseases}

\section{Diet regulation}

Gastrointestinal manifestations in DN mostly perform as different degrees of abnormal gastric emptying because of gastric insufficiency. The diet should be low fat and fibrous, with less residue and fluid-based.

\section{Medication treatment}

There are a variety of drugs for digestive system dysfunction. For gastric insufficiency and obstruction of gastric emptying, a gastric stimulant can be used. For gastrointestinal hemorrhage, PPI and gastric mucosal protectants are available. For diarrhea, there are antidiarrheal agents and somatostatin. For constipation, there are laxatives and probiotics.

\section{Surgical treatment}

Digestive system tumors, such as hepatomas and pancreatic cancer, can be surgically removed after comprehensive evaluation. Cholelithiasis with acute cholecystitis, such as biliary colic, should be treated immediately with emergency surgical treatment, like cholecystectomy and peritoneal drainage. Symptomatic cholecystolithiasis should be treated with cholecystectomy. Oral medication for stone dissolution or extracorporeal shock lithotripsy is recommended if patients refuse surgery.

\section{Other treatments}

FI can be treated with electrical stimulation. Electrical stimulation includes anal electrode electrical stimulation, sacral nerve stimulation, and posterior tibial nerve stimulation. Sacral nerve stimulation involves inserting electrodes into the vicinity of the sacral nerve and continuously pulsating a battery-powered stimulator. For posterior tibial nerve stimulation, a skin surface electrode may be applied to the medial malleolus (percutaneous puncture) above the posterior nerve, or a needle may be inserted into the same area of the skin (percutaneous stimulation).

\section{Prospect}

With the increasing incidence of DM, the population with DN is expanding, and the digestive system symptoms related to DM are attracting more and more attention. Although the exact molecular mechanism of such has not been elucidated, the relevant research is still ongoing. It is possible that in the future, under the circumstances of higher incidence of DN, some digestive system symptoms can be alleviated while treating DN. Grasping the influence of DN on the corresponding parts of the digestive system and studying the relevant mechanisms will be helpful for defining diagnosis and formulating individualized treatment plans.

\section{Conflict of interest}

The authors have no conflict of interests related to this publication.

\section{Author contributions}

Study concept and design (SZ, JM), acquisition of data (SZ), analysis and interpretation of data (SZ), drafting of the manuscript (SZ), critical revision of the manuscript for important intellectual content (SZ, JM), and administrative, technical, or material support, study supervision (JM).

\section{References}

[1] Association AD. 2. Classification and Diagnosis of Diabetes. Diabetes Care 2017;40(Suppl 1):S11. doi:10.2337/dc16-S005.

[2] Flemming NB, Gallo LA, Forbes JM. Mitochondrial dysfunction and signaling in diabetic kidney disease: oxidative stress and beyond. Semin Nephrol 2018;38(2):101-110. doi:10.1016/j.semnephrol.2018.01.001.

[3] Nobuta H, Katagi M, Kume S, Terashima T, Araki SI, Maegawa H, et al. A role for bone marrow-derived cells in diabetic nephropathy. FASEB J 2019;33(3):4067-4076. doi:10.1096/fj.201801825R.

[4] Ihana-Sugiyama N, Nagata N, Yamamoto-Honda R, Izawa E, Kajio H, Shimbo T, et al. Constipation, hard stools, fecal urgency, and incomplete evacuation, but not diarrhea is associated with diabetes and its related factors. World J Gastroenterol 2016;22(11):3252-3260. doi:10.3748/wjg.v22.i11.3252.

[5] Ali R, Raj DS. The gut microbiome, kidney disease, and targeted in terventions. J Am Soc Nephrol 2014;25(4):657-670. doi:10.1681/ ASN.2013080905.

[6] Cerf-Bensussan N, Eberl G. The dialog between microbiota and the immune system: shaping the partners through development and evolution. Semin Immunol 2012;24(1):1-2. doi:10.1016/j. smim.2011.11.007.

[7] Andersen K, Kesper MS, Marschner JA, Konrad L, Ryu M, Kumar Vr $\mathrm{S}$, et al. Intestinal dysbiosis, barrier dysfunction, and bacterial translocation account for CKD-related systemic inflammation. J Am Soc Nephrol 2017;28(1):76-83. doi:10.1681/ASN.2015111285.

[8] Vaziri ND, Yuan J, Norris K. Role of urea in intestinal barrier dysfunction and disruption of epithelial tight junction in chronic kidney disease. Am J Nephrol 2013;37(1):1-6. doi:10.1159/000345969.

[9] Thorens B. GLUT2, glucose sensing and glucose homeostasis. Diabetologia 2015;58(2):221-232. doi:10.1007/s00125-014-3451-1.

[10] Thaiss CA, Levy M, Grosheva I, Zheng D, Soffer E, Blacher E, et al. Hy- 
perglycemia drives intestinal barrier dysfunction and risk for enteric infection. Science 2018;359(6382):1376-1383. doi:10.1126/science. aar3318.

[11] Goto Y, Uematsu S, Kiyono H. Epithelial glycosylation in gut homeostasis and inflammation. Nat Immunol 2016;17(11):1244-1251. doi:10.1038/ni.3587.

[12] Aronwisnewsky J, Clément K. The gut microbiome, diet, and links to cardiometabolic and chronic disorders. Nat Rev Nephrol 2016;12(3):169-181. doi:10.1038/nrneph.2015.191.

[13] Russell WR, Hoyles L, Flint HJ, Dumas ME. Colonic bacterial metabolites and human health. Curr Opin Microbiol 2013;16(3):246-254. doi:10.1016/j.mib.2013.07.002.

[14] Schlee M, Harder J, Köten B, Stange EF, Wehkamp J, Fellermann K. Probiotic lactobacilli and VSL\#3 induce enterocyte beta-defensin 2. Clin Exp Immunol 2008;151(3):528-535. doi:10.1111/j.13652249.2007.03587.x.

[15] Murri M, Leiva I, Gomez-Zumaquero JM, Tinahones FJ, Cardona F, Soriguer F, et al. Gut microbiota in children with type 1 diabetes differs from that in healthy children: a case-control study. BMC Med 2013;11:46. doi:10.1186/1741-7015-11-46.

[16] Lin HV, Frassetto A, Kowalik EJ Jr, Nawrocki AR, Lu MM, Kosinski JR, et al. Butyrate and propionate protect against diet-induced obesity and regulate gut hormones via free fatty acid receptor 3-independent mechanisms. PLoS One 2012;7(4):e35240. doi:10.1371/journal. pone.0035240.

[17] Wichmann A, Allahyar A, Greiner TU, Plovier H, Lundén GÖ, Larsson T, et al. Microbial Modulation of energy availability in the colon regulates intestinal transit. Cell Host Microbe 2013;14(5):582-590. doi:10.1016/j.chom.2013.09.012.

[18] Wong J, Piceno YM, Desantis TZ, Pahl M, Andersen GL, Vaziri ND. Expansion of urease-and uricase-containing, indole-and p-cresolforming and contraction of short-chain fatty acid-producing intestinal microbiota in ESRD. Am J Nephrol 2014;39(3):230-237. doi:10.1159/000360010.

[19] Pellicano R, Ribaldone DG, Fagoonee S, Astegiano M, Saracco GM, Mégraud F. A 2016 panorama of Helicobacter pylori infection: Key messages for clinicians. Panminerva Med 2016;58(4):304-317.

[20] Simon L, Tornóczky J, Tóth $M$, Jámbor M, Sudár $Z$. The significance of Campylobacter pylori infection in gastroenterologic and diabetic practice. Orv Hetil 1989;130(25):1325-1329.

[21] He C, Yang Z, Lu NH. Helicobacter pylori infection and diabetes: is it a myth or fact? World J Gastroenterol 2014;20(16):4607-4617. doi:10.3748/wjg.v20.i16.4607.

[22] Talebi-Taher M, Mashayekhi M, Hashemi MH, Bahrani V. Helicobacter pylori in diabetic and non-diabetic patients with dyspepsia. Acta Med Iran 2012;50(5):315-318.

[23] Zhou F, Zhong X, Chen J, Li C, Shang M, Jiang C, et al. Helicobacter pylori infection associated with type 2 diabetic nephropathy in patients with dyspeptic symptoms. Diabetes Res Clin Pract 2015;110(3):328334. doi:10.1016/j.diabres.2015.09.008.

[24] Tanriverdi O. Association of Helicobacter pylori infection with microalbuminuria in type 2 diabetic patients. Turk J Gastroenterol 2011;22(6):569-574. doi:10.4318/tjg.2011.0252.

[25] Lutsey PL, Pankow JS, Bertoni AG, Szklo M, Folsom AR. Serological evidence of infections and Type 2 diabetes: the MultiEthnic Study of Atherosclerosis. Diabet Med 2009;26(2):149-152. doi:10.1111/ j.1464-5491.2008.02632.x.

[26] Chung GE, Heo NJ, Park MJ, Chung SJ, Kang HY, Kang SJ. Helicobacter pylori seropositivity in diabetic patients is associated with microalbuminuria. World J Gastroenterol 2013;19(1):97-102. doi:10.3748/wjg. v19.i1.97.

[27] Gilbey SG, Walters H, Edmonds ME, Archer AG, Watkins PJ, Parsons $V$, et al. Vascular calcification, autonomic neuropathy, and peripheral blood flow in patients with diabetic nephropathy. Diabet Med 1989;6(1):37-42. doi:10.1111/j.1464-5491.1989.tb01136.x.

[28] Bolli GB, Owens DR. Lixisenatide, a novel GLP-1 receptor agonist: efficacy, safety and clinical implications for type 2 diabetes mellitus. Diabetes Obes Metab 2014;16(7):588-601. doi:10.1111/dom.12253.

[29] Zhang JH, Liu XR, Sheng CX, Liu YE. Analysis on difference in gastrointestinal hormone levels of patients with the history of diabetes and concurrent nephropathy and study on the role of liraglutide. Eur Rev
Med Pharmacol Sci 2017;21(15):3523-3529.

[30] Thomas R, Panackal C, John M, Joshi H, Mathai S, Kattickaran J, et al. Gastrointestinal complications in patients with chronic kidney disease-a 5-year retrospective study from a tertiary referral center. Ren Fail 2013;35(1):49-55. doi:10.3109/0886022X.2012.731998.

[31] Jia Y, Guo Z. Recent advances in the pathogenesis and treatment of digestive system symptoms in chronic renal failure. Chinese Journal of Integrated Chinese and Western Medicine Nephropathy 2014(10):936-937.

[32] Maev IV, lurenev GL, Kakhramanova DA. Peculiarities of gastroesophageal reflux disease in patients with type 2 diabetes mellitus. Klin Med (Mosk) 2012;90(9):53-56.

[33] Camilleri M, Chedid V, Ford AC, Haruma K, Horowitz M, Jones KL, et al. Gastroparesis. Nat Rev Dis Primers 2018;4(1):41. doi:10.1038/ s41572-018-0038-z.

[34] Kofod-Andersen K, Tarnow L. Prevalence of gastroparesis-related symptoms in an unselected cohort of patients with Type 1 diabetes. J Diabetes Complications 2012;26(2):89-93. doi:10.1016/j.jdiacomp.2012.02.009.

[35] Bharucha AE, Batey-Schaefer B, Cleary PA, Murray JA, Cowie C, Lorenzi $\mathrm{G}$, et al. Delayed gastric emptying is associated with early and long-term hyperglycemia in type 1 diabetes mellitus. Gastroenterology 2015;149(2):330-339. doi:10.1053/j.gastro.2015.05.007.

[36] Chen H, Ning L, Cheng L, Liu E, Guo H. Use of different drugs and the risk of upper gastrointestinal bleeding: a case-control study. Journal of Pharmacoepidemiology 2016(4):239-244.

[37] Chang F, Zhang L, Meng M. Causes of death in 50 patients with uremia undergoing hemodialysis. Modern Diagnosis and Treatment 2013(11):2504-2505.

[38] Syam AF, Simadibrata M, Wanandi SI, Hernowo BS, Sadikin M, Rani AA. Gastric ulcers induced by systemic hypoxia. Acta Med Indones 2011;43(4):243-248.

[39] Zhong HJ, Yuan Y, Xie WR, Chen MH, He XX. Type 2 diabetes mellitus is associated with more serious small intestinal mucosal injuries. PLoS One 2016;11(9):e0162354. doi:10.1371/journal.pone.0162354.

[40] Cheung CY, Ma MKM, Chak WL, Tang SCW. Cancer risk in patients with diabetic nephropathy: A retrospective cohort study in Hong Kong. Medicine (Baltimore) 2017;96(38):e8077. doi:10.1097/ MD.0000000000008077.

[41] Gordon-Dseagu VL, Shelton N, Mindell JS. Epidemiological evidence of a relationship between type- 1 diabetes mellitus and cancer: $A$ review of the existing literature. Int J Cancer 2013;132(3):501-508. doi:10.1002/ijc.27703.

[42] Hardt PD, Erbaeh M, Schnell O. Exocrine pancreatic insufficiency and diabetes. MMW Fortschr Med 2012;154:53-54.

[43] Xiang $X$, Zhu H. Pathogenesis and treatment of diabetic diarrhea. Int J Endocrinol Metab 2013;33(2):92-95. doi:10.3760/cma.j.is sn.1673-4157.2013.02.006.

[44] D’Addio F, La Rosa S, Maestroni A, Jung P, Orsenigo E, Ben Nasr M, et al. Circulating IGF-I and IGFBP3 levels control human colonic stem cell function and are disrupted in diabetic enteropathy. Cell Stem Cell 2015;17(4):486-498. doi:10.1016/j.stem.2015.07.010.

[45] Bharucha AE, Dunivan G, Goode PS, Lukacz ES, Markland AD, Matthews CA, et al. Epidemiology, pathophysiology, and classification of fecal incontinence: state of the science summary for the national institute of diabetes and digestive and kidney diseases (NIDDK) Workshop. Am J Gastroenterol 2015;110(1):127-136. doi:10.1038/ ajg.2014.396.

[46] Drossman DA, Hasler WL. Rome IV-functional GI disorders: disorders of gut-brain interaction. Gastroenterology 2016;150(6):1257-1261. doi:10.1053/j.gastro.2016.03.035.

[47] Tseng PH, Lee YC, Chiu HM, Chen CC, Liao WC, Tu CH, et al. Association of diabetes and $\mathrm{HbA} 1 \mathrm{c}$ levels with gastrointestinal manifestations. Diabetes Care 2012;35(5):1053-1060. doi:10.2337/dc111596.

[48] Cusi K. Role of obesity and lipotoxicity in the development of nonalcoholic steatohepatitis: pathophysiology and clinical implications. Gastroenterology 2012;142(4):711-725.e6. doi:10.1053/j.gastro.2012.02.003.

[49] Bril F, Sninsky JJ, Baca AM, Superko HR, Portillo Sanchez P, Biernacki $D$, et al. Hepatic steatosis and insulin resistance, but not steatohepa- 
titis, promote atherogenic dyslipidemia in NAFLD. J Clin Endocrinol Metab 2016;101(2):644-652. doi:10.1210/jc.2015-3111.

[50] Targher G, Bertolini L, Chonchol M, Rodella S, Zoppini G, Lippi G, et al. Non-alcoholic fatty liver disease is independently associated with an increased prevalence of chronic kidney disease and retinopathy in type 1 diabetic patients. Diabetologia 2010;53(7):1341-1348 doi:10.1007/s00125-010-1720-1.

[51] Milic S, Stimac D. Nonalcoholic fatty liver disease/steatohepatitis: epidemiology, pathogenesis, clinical presentation and treatment. Dig Dis 2012;30(2):158-162. doi:10.1159/000336669.

[52] Jenks SJ, Conway BR, Hor TJ, Williamson RM, McLachlan S, Robertson $C$, et al. Hepatic steatosis and non-alcoholic fatty liver disease are not associated with decline in renal function in people with Type 2 diabetes. Diabet Med 2014;31(9):1039-1046. doi:10.1111/dme.12456.

[53] Targher G, Bertolini L, Rodella S, Lippi G, Zoppini G, Chonchol M. Relationship between kidney function and liver histology in subjects with nonalcoholic steatohepatitis. Clin J Am Soc Nephrol 2010;5(12):2166-2171. doi:10.2215/CJN.05050610.

[54] Wijarnpreecha K, Thongprayoon C, Boonpheng B, Panjawatanan $P$, Sharma K, Ungprasert $P$, et al. Nonalcoholic fatty liver disease and albuminuria: a systematic review and meta-analysis. Eur J Gastroenterol Hepatol 2018;Sep30(9):986-994. doi:10.1097/ MEG.0000000000001169.

[55] Yeung MW, Wong GL, Choi KC, Luk AO, Kwok R, Shu SS, et al. Advanced liver fibrosis but not steatosis is independently associated with albuminuria in Chinese patients with type 2 diabetes. J Hepato 2018;68(1):147-156. doi:10.1016/j.jhep.2017.09.020.

[56] Bril F, Cusi K. Management of nonalcoholic fatty liver disease in patients with type 2 diabetes: a call to action. Diabetes Care 2017;40(3):419-430. doi:10.2337/dc16-1787.

[57] Nevalainen PI, Kallio T, Lahtela JT, Mustonen J, Pasternack AI. High peritoneal permeability predisposes to hepatic steatosis in diabetic continuous ambulatory peritoneal dialysis patients receiving intraperitoneal insulin. Perit Dial Int 2000;20(6):637-642.

[58] Zhan YT, Zhang C, Li L, Bi CS, Song X, Zhang ST. Non-alcoholic fatty liver disease is not related to the incidence of diabetic nephropathy in type 2 diabetes. Int J Mol Sci 2012;13(11):14698-14706. doi:10.3390/ijms131114698.

[59] Wang C, Wang X, Gong G, Ben Q, Qiu W, Chen Y, et al. Increased risk of hepatocellular carcinoma in patients with diabetes mellitus: $A$ systematic review and meta-analysis of cohort studies. Int J Cancer 2012;130(7):1639-1648. doi:10.1002/ijc.26165.

[60] Leite NC, Villela-Nogueira CA, Pannain VL, Bottino AC, Rezende GF, Cardoso CR, et al. Histopathological stages of nonalcoholic fatty liver disease in type 2 diabetes: prevalences and correlated factors. Liver Int 2011;31(5):700-706. doi:10.1111/j.1478-3231.2011.02482.x.

[61] Weng CJ, Hsieh YH, Tsai CM, Chu YH, Ueng KC, Liu YF, et al. Relationship of insulin-like growth factors system gene polymorphisms with the susceptibility and pathological development of hepatocellular carcinoma. Ann Surg Oncol 2010;17(7):1808-1815. doi:10.1245/ s10434-009-0904-8.

[62] Targher G, Bertolini L, Rodella S, Zoppini G, Lippi G, Day C, et al. Non-alcoholic fatty liver disease is independently associated with an increased prevalence of chronic kidney disease and proliferative/ laser-treated retinopathy in type 2 diabetic patients. Diabetologia 2008;51:444-450. doi:10.1007/s00125-007-0897-4

[63] Xiang $X$, Zhu H. Progress in the pathogenesis of diabetes complicated with gallstones. Int J Dig Dis 2013;33(6):389-391. doi:10.3969/j. issn.1673-534X.2013.06.010.

[64] Chen L, Zhang XF, Ku BQ, Wang XC, Ma C, Liang JY, et al. Effects of acupoint injection of autologous blood on symptoms and plasma motilin and gastrin levels of diabetic gastroparesis patients. Zhen Ci Yan Jiu 2012;37(3):229-232246.
[65] Agunloye AM, Adebakin AM, Adeleye JO, Ogunseyinde AO. UItrasound preyalence of gallstone disease in diabetic patients at Ibadan, Nigeria.Niger J Clin Pract. 2013;16:71-75. doi:10.4103/11193077.106770 .

[66] Toosi FS, Ehsanbakhsh AR, Tavakoli MR. Asymptomatic gallstones and related risk factors in Iran. Hepatogastroenterology 2011;58(109):1123-1126. doi:10.5754/hge11060.

[67] Larger E, Philippe MF, Barbot-Trystram L, Radu A, Rotariu M, Nobécourt $E$, et al. Pancreatic exocrine function in patients with diabetes. Diabet Med 2012;29(8):1047-1054. doi:10.1111/j.14645491.2012.03597.x.

[68] Weitgasser R, Abrahamian H, Clodi M, Fortunat W, Hammer H. Position paper: exocrine pancreatic insufficiency and diabetes mellitus. Wien Klin Wochenschr 2012;124(Suppl 2):100-103. doi:10.1007/ s00508-012-0290-2.

[69] Han J, Liu YQ. Suppressed glucose metabolism in acinar cells might contribute to the development of exocrine pancreatic insuffleiency in streptozotocin-induced diabetic mice. Metabolism 2010;59(9):12571267. doi:10.1016/j.metabol.2009.11.018.

[70] Urushihara H, Taketsuna M, Liu Y, Oda E, Nakamura M, Nishiuma S, et al. Increased risk of acute pancreatitis in patients with type 2 diabetes: an observational study using a Japanese hospital database. PLoS One 2012;7(12):e53224. doi:10.1371/journal.pone.0053224.

[71] Andersen DK, Andren-Sandberg Å, Duell EJ, Goggins M, Korc M, Petersen GM, et al. Pancreatitis-diabetes-pancreatic cancer: summary of an NIDDK-NCI workshop. Pancreas 2013;42(8):1227-1237. doi:10.1097/MPA.0b013e3182a9ad9d.

[72] Aggarwal G, Rabe KG, Petersen GM, Chari ST. New-onset diabetes in pancreatic cancer: A study in the primary care setting. Pancreatology 2012;12(2):156-161. doi:10.1016/j.pan.2012.02.003.

[73] Er KC, Hsu CY, Lee YK, Huang MY, Su YC. Effect of glycemic control on the risk of pancreatic cancer: A nationwide cohort study. Medicine (Baltimore) 2016;95(24):e3921. doi:10.1097/MD.0000000000003921.

[74] Lu Y, García Rodríguez LA, Malgerud L, González-Pérez A, Martín-Pérez $\mathrm{M}$, Lagergren J, et al. New-onset type 2 diabetes, elevated $\mathrm{HbA} 1 \mathrm{c}$ anti-diabetic medications, and risk of pancreatic cancer. Br J Cancer 2015;113(11):1607-1614. doi:10.1038/bjc.2015.353.

[75] Rahman A. Type 2 diabetes and of risk of pancreatic adenocarcinoma. Lancet Oncol 2014;15(10):e420. doi:10.1016/S1470-2045(14)70368-

[76] Aoki K, Kamiyama H, Masuda K, Togashi Y, Terauchi Y. Mosapride citrate, a 5-HT4 receptor agonist, increased the plasma active and total glucagon-like peptide-1 levels in non-diabetic men. Endocr 2013;60(4):493-499. doi:10.1507/endocrj.EJ12-0350.

[77] Zhang JH, Liu XR, Sheng CX, Liu YE. Analysis on difference in gastrointestinal hormone levels of patients with the history of diabetes and concurrent nephropathy and study on the role of liraglutide. Eur Rev Med Pharmacol Sci 2017;21(15):3523-3529.

[78] Gómez-Izquierdo JC, Yu OHY. The influence of proton-pump inhibitors on glycemic control: a systematic review of the literature and a meta-analysis. Can J Diabetes 2017;41(4):351-361. doi:10.1016/j. jcjd.2016.11.004

[79] Takebayashi K. Effect of proton pump inhibitors on glycemic contro in patients with diabetes. World J Diabetes 2015;6(10):1122-1131. doi:10.4239/wjd.v6.i10.1122.

[80] Antoniou T, Macdonald EM, Hollands S, Gomes T, Mamdani MM, Garg $A X$, et al. Proton pump inhibitors and the risk of acute kidney injury in older patients: a population-based cohort study. CMAJ Open 2015;3(2):E166-E171. doi:10.9778/cmajo.20140074.

[81] Leussink BT, Nagelkerke JF, van de Water B, Slikkerveer A, van der Voet GB, Srinivasan A, et al. Pathways of Proximal Tubular Cell Death in Bismuth Nephrotoxicity. Toxicol Appl Pharmacol 2002;180(2):100109. doi:10.1006/taap.2002.9379. 